\title{
Communication \\ Application of a Novel Nd:YAG/PPMgLN Laser Module Speckle-Suppressed by Multi-Mode Fibers in an Exhibition Environment
}

\author{
Xiao Liu ${ }^{1}$, Xin-Ting Zeng ${ }^{1}$, Wen-Jian Shi ${ }^{2}$, Shang-Feng Bao ${ }^{3}$, Tao Yu ${ }^{3}$ and Hong-Yi Lin ${ }^{3, *(D)}$ \\ 1 School of Cultural Industries and Tourism, Xiamen University of Technology, Xiamen 361024, China; \\ xiaoanneliu@163.com (X.L.); 2011110401@xmut.edu.cn (X.-T.Z.) \\ 2 Fujian Zoomking Technology Co., Ltd., Longyan 364100, China; xiamenboy@126.com \\ 3 Fujian Key Laboratory of Optoelectronic Technology and Devices, Xiamen University of Technology, \\ Xiamen 361024, China; 2122031292@stu.xmut.edu.cn (S.-F.B.); opolaser@163.com (T.Y.) \\ * Correspondence: linyi0714@163.com
}

check for updates

Citation: Liu, X.; Zeng, X.-T.; Shi, W.-J.; Bao, S.-F.; Yu, T.; Lin, H.-Y. Application of a Novel Nd:YAG/PPMgLN Laser Module Speckle-Suppressed by Multi-Mode Fibers in an Exhibition Environment. Photonics 2022, 9, 46. https:// doi.org/10.3390/photonics9010046

Received: 27 December 2021

Accepted: 14 January 2022

Published: 16 January 2022

Publisher's Note: MDPI stays neutral with regard to jurisdictional claims in published maps and institutional affiliations.

Copyright: (C) 2022 by the authors. Licensee MDPI, Basel, Switzerland. This article is an open access article distributed under the terms and conditions of the Creative Commons Attribution (CC BY) license (https:// creativecommons.org/licenses/by/ $4.0 /)$.

\begin{abstract}
Laser exhibition technology has been widely used in the virtual environment of exhibitions and shows, as well as in the physical conference and exhibition centers. However, the speckle issue due to the high coherence of laser sources has caused harmful impacts on image quality, which is one of the obstacles to exhibition effects. In this paper, we design a compact Nd:YAG/PPMgLN laser module at $561.5 \mathrm{~nm}$ and use two different types of big-core multi-mode fibers to lower the spatial coherence. According to our experiment, the speckle contrasts relating to these two types reduce to $7.9 \%$ and $4.1 \%$, respectively. The results of this paper contribute to improving the application effects of key optical components in the exhibitions. Only in this way can we provide technical supports and service guarantee for the development of the exhibition activities, and an immersive interactive experience for the audiences.
\end{abstract}

Keywords: exhibitions; laser; speckle suppression; fiber; Nd:YAG/PPMgLN crystal

\section{Introduction}

With the rapid development of information and communication technologies (ICTs), audiences' demands for audio-visual effects have gradually increased. This has led to obvious shifts in product content, key technologies, business models and application scenarios in the exhibition industry [1,2]. According to current studies, the requirements of exhibitions and shows cannot be met adequately by means of traditional photo-electric technologies while modern forms of exhibitions and shows have made some headway by virtue of optical techniques, computer techniques, landscape design and art media [3,4]. Laser exhibition technology combines exhibition scenes with virtual illusions and reality by means of the coordination and adjustment of light, which provides a more attractive, stunning and realistic effect for the audiences on the spot [5]. Laser exhibition technology integrates holographic techniques and gesture control technology, realizing the interaction of humans and exhibits and audience-stereoscopic images [6,7].

Under the influence of laser technologies, breakthroughs in artistic exhibitions have been achieved. However, there still remain key technological issues to be solved, such as laser sources, optical coupling, laser speckles, as well as color restoration and correction $[8,9]$. In this paper, we design a novel Nd:YAG/PPMgLN laser source module at $561.5 \mathrm{~nm}$ and use two different types of big-core multi-mode fibers to suppress the speckle effect, in order to achieve a better exhibition effect for visitors and audiences and promote the development of the conference and exhibition industry. 


\section{Nd:YAG/PPMgLN Module at $561.5 \mathrm{~nm}$}

\subsection{Properties of the Nd:YAG/PPMgLN Module}

The PPMgLN crystal is one of the most effective non-linear media, which can turn a nearinfrared fundamental laser into visible light based on the double-frequency method [10-13]. The physical picture and the schematic drawing of the Nd:YAG/PPMgLN module at $561.5 \mathrm{~nm}$ are shown in Figure 1. This module is very simple, compact and efficient [14]. Here, the novel module consists of a PPMgLN crystal, an Nd:YAG gain medium, a silicon wafer, and an aluminum housing. The well-designed coating of this module directly adheres to the facets of the Nd:YAG/PPMgLN crystal. The length of the Nd:YAG crystal is $3 \mathrm{~mm}$. The left facet $\left(\mathrm{M}_{\mathrm{in}}\right)$ of the Nd:YAG crystal has a high-reflection $(\mathrm{HR})$ coating at $1123.0 \mathrm{~nm}$ and a high-transmittance (HT) coating at 808.0, 1064.0 and $1319.0 \mathrm{~nm}$. The right facet has an HR coating at $561.5 \mathrm{~nm}$ and an HT coating at 946.0, 1064.0, 1123.0 and $1319.0 \mathrm{~nm}$.
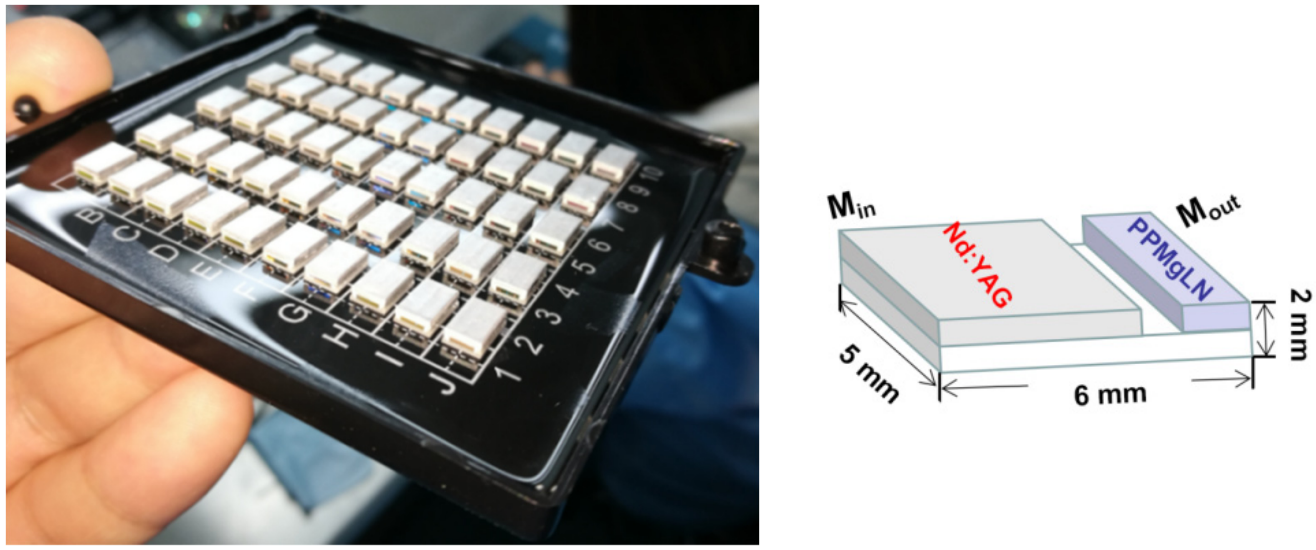

Figure 1. The physical picture (left) and the schematic drawing (right) of the Nd:YAG/PPMgLN module.

The length of the PPMgLN crystal is $2 \mathrm{~mm}$. The left facet of the PPMgLN crystal has an HT coating at 561.5, 946.0, 1064.0, 1123.0 and $1319.0 \mathrm{~nm}$. The right facet $\left(\mathrm{M}_{\text {out }}\right)$ has an HR coating at $1123.0 \mathrm{~nm}$ and an HT coating at 561.5, 946.0, 1064.0 and $1319.0 \mathrm{~nm}$. The module is directly pumped by a $3 \mathrm{~W} c$-mount LD, and the output power at $561.5 \mathrm{~nm}$ is $250 \mathrm{~mW}$ at the pump power of $2.5 \mathrm{~W}$ with the conversion efficiency of $10 \%$.

\subsection{Spectral Characteristic of the Nd:YAG/PPMgLN Laser}

Spectral characteristics are the key parameter for speckle analysis. In theory, for the constant fundamental light and the plane wave, the doubled-frequency efficiency $\eta$ can be written as:

$$
\eta \propto \sin c^{2}\left(\frac{\Delta k L}{2}\right)
$$

$L$ is the length of the PPMgLN crystal. $\Delta k$ is the phase mismatch factor for quasi-phasematching, which is written as:

$$
\Delta k=2 \pi\left[\frac{n\left(\lambda_{2 \omega}, T\right)}{\lambda_{2 \omega}}-\frac{n\left(\lambda_{\omega}, T\right)}{\lambda_{\omega}}-\frac{1}{\Lambda}\right]
$$

$\lambda_{\omega}$ and $\lambda_{2 \omega}$ are the wavelengths of the fundamental light and frequency-doubled light. $n, \Lambda$ and $T$ are the refractive index, period and temperature of the PPMgLN crystal, respectively. The Sellmeier equation for the PPMgLN crystal [15] is:

$$
n^{2}(\lambda, T)=5.319725+4.753496 \times 10^{-7} f+\frac{0.09147285+3.310965 \times 10^{-8} f}{\lambda^{2}-0.3165008^{2}}+\frac{100.2028+2.760513 \times 10^{-5} f}{\lambda^{2}-11.37639^{2}}-1.497046 \times 10^{-2} \lambda^{2}
$$


$f$ is the temperature parameter, and it can be described as follows:

$$
f=(T-24.5)(T+470.82)
$$

When the PPMgLN length is $2 \mathrm{~mm}$, the direct relationship between the normalized efficiency and the fundamental wavelength is calculated and is shown in Figure 2. The black line represents the theoretical calculation of the normalized efficiency versus the fundamental wavelength. The red line is the actually measured spectrum of the fundamental light. From the viewpoint of theory, the acceptance band-width at $1123.0 \mathrm{~nm}$ is $1.2 \mathrm{~nm}$, i.e., 1122.4-1123.6 nm. Therefore, the spectral line-width of the frequency-doubled light at $561.5 \mathrm{~nm}$ is $0.6 \mathrm{~nm}$, i.e., $561.2-561.8 \mathrm{~nm}$.

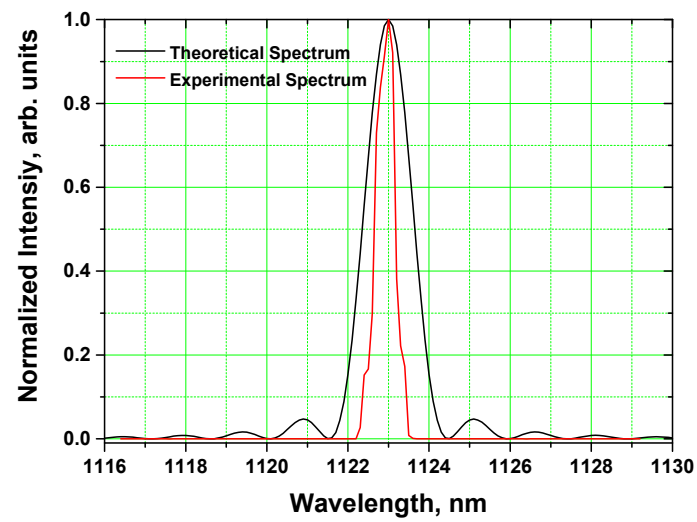

Figure 2. The normalized efficiency versus the fundamental wavelength (black line) and the actually measured spectrum of the fundamental light (red line).

In our experiment, the actual fundamental line-width at $1123.0 \mathrm{~nm}$ is only $0.6 \mathrm{~nm}$, i.e., $1122.7-1123.3 \mathrm{~nm}$. Therefore, the corresponding line-width at $561.5 \mathrm{~nm}$ is only $0.3 \mathrm{~nm}$, i.e., $561.35-561.65 \mathrm{~nm}$. The coherence time $\left(\tau_{\mathcal{c}}\right)$ and coherence length $\left(L_{\mathcal{C}}\right)$ at $561.5 \mathrm{~nm}$ can be written as:

$$
\begin{gathered}
L_{c}=\frac{\lambda_{0}^{2}}{\Delta \lambda} \\
\tau_{c}=\frac{L_{c}}{c}=\frac{\lambda_{0}^{2}}{\Delta \lambda} \frac{1}{c}
\end{gathered}
$$

$c, \lambda_{0}$ and $\Delta \lambda$ are the speed of light, the wavelength and the spectral line-width at $561.5 \mathrm{~nm}$ in a vacuum, respectively. The coherence length and coherence time at $561.5 \mathrm{~nm}$ are $1.05 \mathrm{~mm}$ and $3.5 \mathrm{ps}$, respectively. Here, the fundamental wavelength is fixed, so it is not easy to widen the line-width at $561.5 \mathrm{~nm}$ with only one PPMgLN crystal to decrease the temporal coherence [16].

\section{Laser Speckle}

When the Nd:YAG/PPMgLN laser beam at $561.5 \mathrm{~nm}$ irradiates an imaging plate, uneven intensity distribution can be shown in Figure 3. The bright spots are the interference enhancement of diffuse reflections in each small rough facet of the imaging plate, while the dark spots are the destructive interference of coherent light. We obtain the laser illumination image by a CCD camera and its 3D intensity distribution is analyzed by MATLAB. The standard deviation and the mean value of the illumination image can be calculated, and their ratio reflects the speckle contrast. Speckle contrast is $46.2 \%$. Obviously, the light intensity distribution is very uneven. The relative intensity ratio has changed from 25 to 250 , and the difference ratio is 225 . 

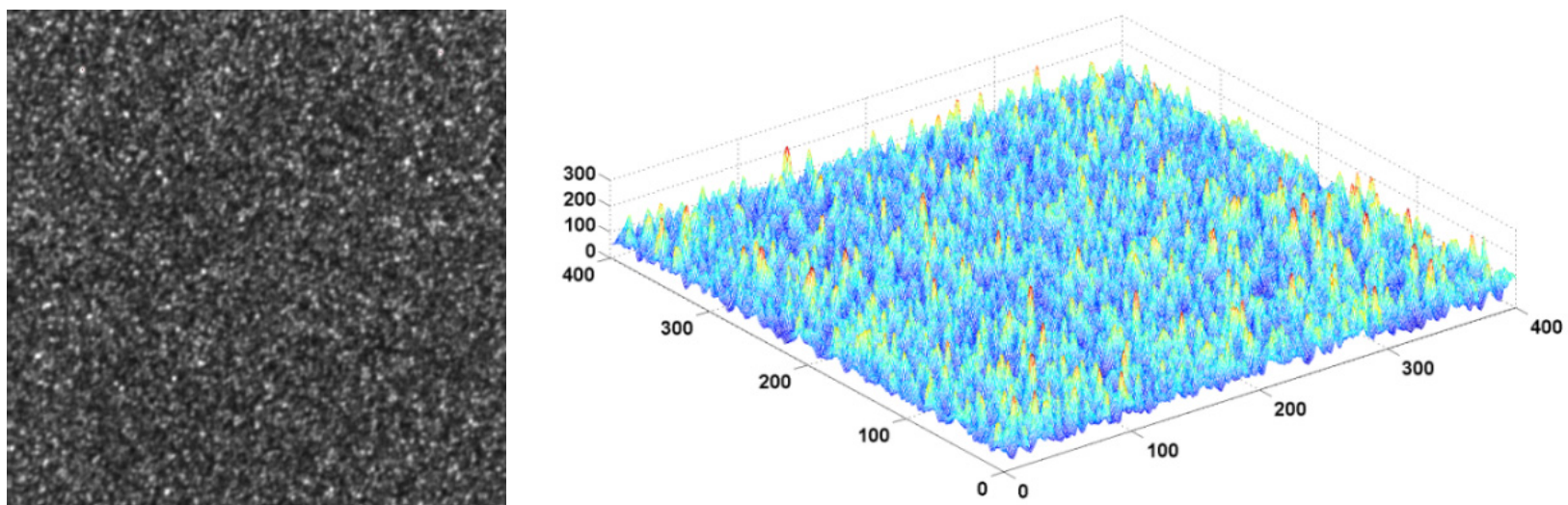

Figure 3. A laser illumination image is recorded by a CCD camera with the speckle contrast of $46.2 \%$ (left) and 3D intensity distribution is analyzed by MATLAB (right).

In the field of laser exhibitions, speckle can lead to the partial loss of image information and the reduction of image resolution [17]. In order to improve the visitors' experience, it is necessary to suppress the laser speckle by means of laser technology. There are three ways to eliminate the laser speckle: (a) by reducing the temporal coherence; (b) by reducing spatial coherence; and (c) by dynamic superposing multiple images [18,19]. In 2016, Z. Xin reported an efficient, wide line-width and speckle-reduced green output by second harmonic generation with a multiple LBO crystal cascade [16]. When three-crystal cascade was used, the acceptance band-width at $1064 \mathrm{~nm}$ reaches $8 \mathrm{~nm}$ with a high conversion efficiency of $30.5 \%$. The wider line-width of the green laser can further reduce the temporal coherence and eliminate the laser speckle.

The introduction of a multi-mode fiber is also an effective method to weaken laser speckle. It is also beneficial to laser transmission with less energy loss [20-22]. By meticulously changing the core diameter and the numerical aperture of the gradient-index fiber, the phase differences between each mode may vary randomly over time. Therefore, the "boiling" speckle pattern also alters over time and superimposes on the receiving screen [23]. Due to vision persistence of human eyes, the intensity superposition of multiple speckle patterns can reduce the speckle contrast effectively. In 2019, Y. Yoon used a diamond/KBr diffuser coupled with a multi-mode fiber to reduce the speckle noise of a tunable external cavity quantum cascade lasers [24]. In this paper, we use two different types of big-core multi-mode fibers to lower the spatial coherence of the Nd:YAG/PPMgLN laser at $561.5 \mathrm{~nm}$.

\section{Speckle-Suppressed Technology Based on Multi-Mode Fibers}

\subsection{Experimental Setup of Speckle-Suppressed Technology Based on Multi-Mode Fibers}

We employ two types of big-core multi-mode fibers to reduce speckle contrast. The experimental setup includes an Nd:YAG/PPMgLN laser at $561.5 \mathrm{~nm}$, a coupling lens, a multi-mode fiber, an imaging plate and a CCD camera (FT-CS140H, Fortune Techno. Ltd., Shenzhen, China) as shown in Figure 4. The laser at $561.5 \mathrm{~nm}$ is coupled in the multi-mode fiber by the coupling lens and then illuminates the white imagining plate. We choose SUH500 multi-mode quartz fibers and a 1000/2200 multi-mode plastic fiber for our experiment. The laser illumination image is detected by the CCD camera, and 3D intensity distribution is analyzed by MATLAB.

We can use the inter-modal dispersion of the multi-mode gradient-index fiber to reduce spatial coherence, with its schematic diagram shown in Figure 5. Moreover, the multi-mode fiber can disturb the polarization characteristics of the propagating light, which contributes to suppressing the laser speckle. 


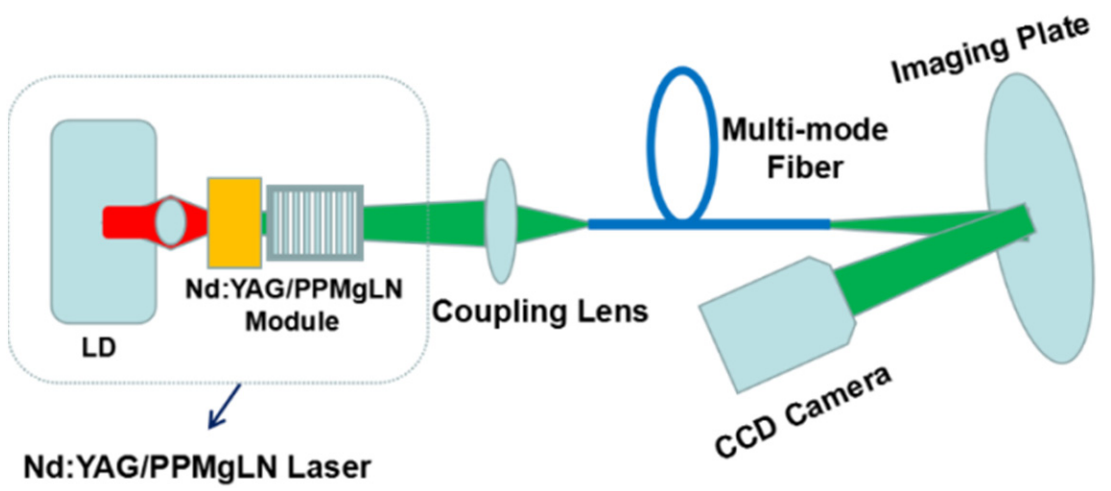

Figure 4. A speckle-suppressed Nd:YAG/PPMgLN laser at $561.5 \mathrm{~nm}$ based on a big-core multimode fiber.

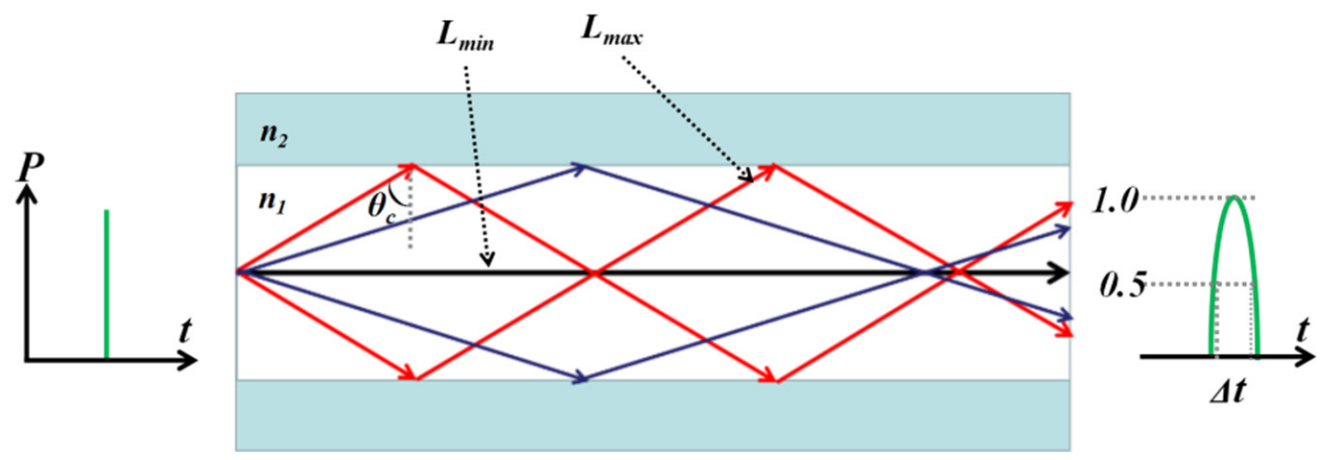

Figure 5. The inter-modal dispersion of the multi-mode gradient-index fiber.

\subsection{The Dispersion Characteristics of the Multi-Mode Fibers}

According to the selected fiber parameters, such as the core diameter $(d)$, the numerical aperture $(N A)$ and the wavelength of the transmitted light $\left(\lambda_{0}\right)$, the number of propagation modes $(N)$ of the multi-mode fiber is written as:

$$
N \approx \frac{\pi d}{\lambda_{0}}(N A)
$$

The number of modes $(N)$ is proportional to the core diameter $(d)$ and the numerical aperture $(N A)$. For the multi-mode quartz fiber, the transmitted wavelength and the numerical aperture are $561.5 \mathrm{~nm}$ and 0.2 , respectively. When the core diameters are 50, 100, 200,300 and $475 \mu \mathrm{m}$, respectively, the numbers of corresponding modes are 56, 112, 224, 336 and 531. For the multi-mode plastic fiber, the numerical aperture is 0.5 and the core diameter is $1000 \mu \mathrm{m}$. Then, the final result for the number of modes is 2797 . Obviously, the bigger the core diameter and the numerical aperture, the greater the number of fiber modes as well as the greater the dispersion.

Furthermore, the propagation delay time $(\Delta t)$ of the multi-mode fiber can be written as:

$$
\Delta t=\frac{n_{1}\left(L_{\max }-L_{\min }\right)}{c} \approx \frac{L_{\min }}{2 c n_{1}}(N A)^{2}=\frac{L}{2 c n_{1}}(N A)^{2}
$$

$L$ is the length of the multi-mode fiber. $L_{\max }$ and $L_{\min }$ are the maximum propagation distance and the minimum propagation distance between the different modes of the multimode fiber. Formula (8) shows that delay time $(\Delta t)$ is proportional to the fiber length $(L)$ and the square of numerical aperture $(N A)^{2}$. The longer the $L$ is, the larger the $N A$, as well as the larger the time difference $(\Delta t)$ between multiple communication modes. For the multi-mode plastic fiber with the numerical aperture of 0.5 and the core refractive index of 1.516, the delay time reaches $275 \mathrm{ps}$. However, for the multi-mode quartz fiber with the numerical aperture of 0.2 and the core refractive index of 1.450 , the delay time is only 46 ps. 
Every delay time $(\Delta t)$ of these multi-mode fibers is longer than the coherence time $\left(\tau_{c}\right)$ of the Nd:YAG/PPMgLN laser at $561.5 \mathrm{~nm}$, and these multi-mode fibers can be used to lower the spatial coherence.

\section{Results and Discussion}

\subsection{Multi-Mode Quartz Fibers}

The aforementioned SUH500 multi-mode quartz fibers with five different cores are used. Their core diameters are 50,100, 200, 300 and $475 \mu \mathrm{m}$, respectively, with the same length of $1 \mathrm{~m}$. The speckle contrast ratios versus the core diameters of the quartz fibers are shown in Figure 6. The speckle contrast ratios are 26.4\%, 19.2\%, 14.2\%, 10.9\% and $7.9 \%$, when the mode numbers of these multi-mode fibers are 56, 112, 224, 336 and 531, respectively. The growth of the core diameter and mode number can greatly decrease the speckle contrast.

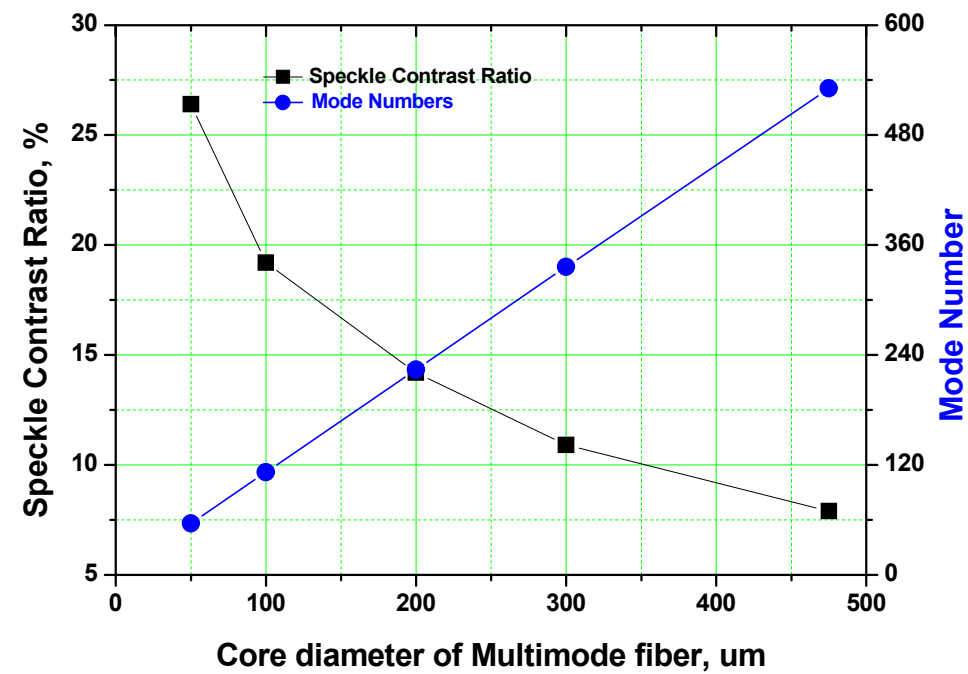

Figure 6. The speckle contrast ratios and mode numbers versus the core diameters of the multi-mode quartz fiber.

In the integration time of the CCD camera, the superpositions of some speckle structures constituted by the corresponding different fiber modes could weaken the speckle contrast to a certain extent. The specific weakening degree is determined by the relationship among the speckle structures of all the fiber modes. When these speckle structures are independent of one another, the speckle contrast could decrease significantly. When these speckle structures are completely coherent, the contrast is the same as that in free space. Among these quartz fibers, the 475/500 SUH500 multi-mode fiber possesses the largest mode number of 531, so it reveals the maximum incoherent superpositions with the lowest speckle contrast ratio of $7.9 \%$.

The laser illumination image and 3D intensity distribution of the 475/500 SUH500 multi-mode fiber are shown in Figure 7 . The speckle is very obvious before passing the 475/500 SUH500 fiber, however, the intensity distribution homogenizes with the speckle contrast reduction of $7.9 \%$ after passing the multi-mode fiber. The value range of relative strength is 80 , i.e., $160-240$. The power at $561.5 \mathrm{~nm}$ is $233 \mathrm{~mW}$ with the total coupling efficiency of $93 \%$. 

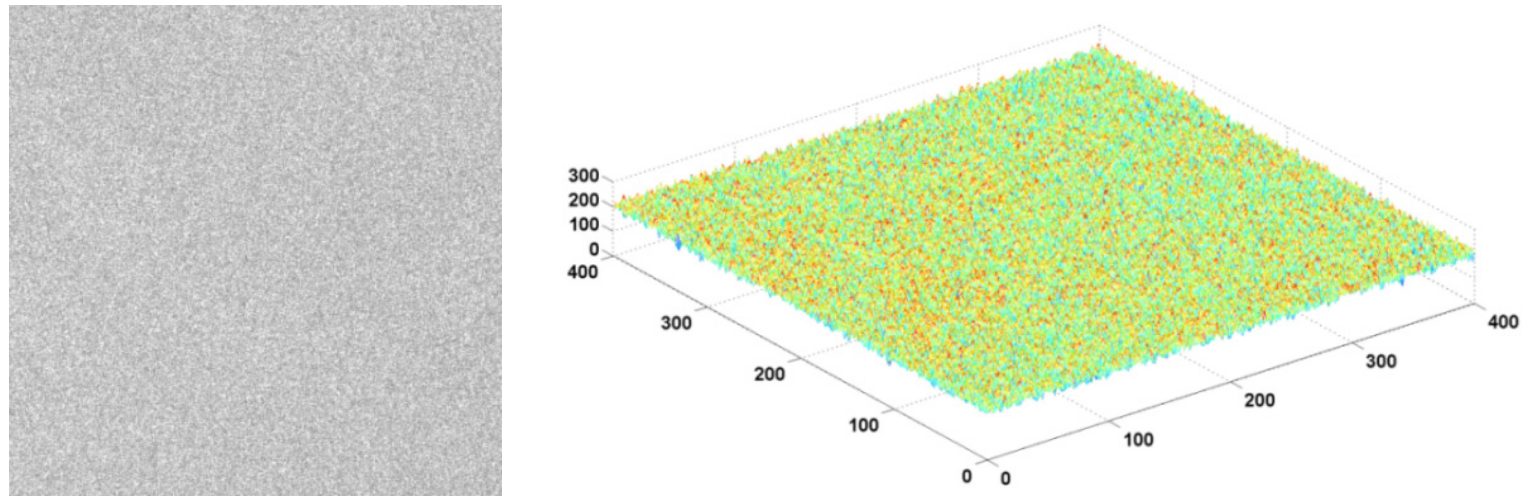

Figure 7. A laser illumination image with the speckle contrast of $7.9 \%$ (left) and 3D intensity distribution (right) based on a speckle-reduced 475/500 SUH500 multi-mode fiber.

\subsection{Multi-Mode Plastic Fiber}

The 1000/2200 multi-mode plastic fiber is also used to further reduce the laser speckle. The intensity distribution becomes better-distributed after passing the plastic fiber, and the speckle contrast reduces to $4.1 \%$. The range of relative strength value is 50 , i.e., $180-230$ in Figure 8. Moreover, the power at $561.5 \mathrm{~nm}$ is $223 \mathrm{~mW}$ with the total coupling efficiency of $89 \%$.
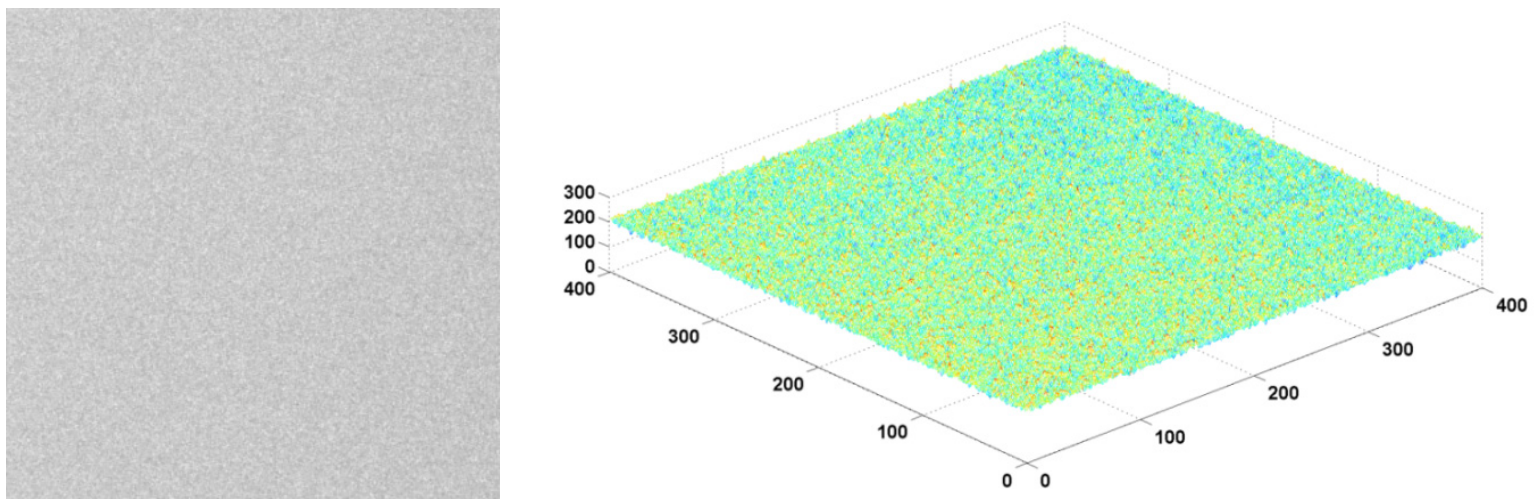

Figure 8. A laser illumination image (left) with the speckle contrast of $4.1 \%$ and $3 \mathrm{D}$ intensity distribution (right) based on a speckle-suppressed 1000/2200 multi-mode plastic fiber.

The multi-mode plastic fiber has the maximum mode number of 2797 and the longest propagation delay time of $275 \mathrm{ps}$. Therefore, the speckle-suppressed effect is the most obvious. When the delay time of multiple modes $(\Delta t)$ is more than the coherence time $\left(\tau_{c}\right)$ of the spectrum, that is to say, optical path difference between adjacent modes exceeds coherence length $\left(L_{c}\right)$, more temporal coherence could be weakened significantly. Furthermore, the longer the $L$ is, the larger the $N A$, as well as the greater the reduction of the temporal coherence. Therefore, the more independent speckle patterns that are obtained, the more obvious is the speckle contrast reduction.

The $561.5 \mathrm{~nm}$ laser is used as the illumination light to make a comparison in the display images of a resolution test target between speckle pre-suppression and speckle post-suppression in Figure 9. It can be seen that the resolution of the image (left) is very poor due to the influence of speckle. However, because of the inter-modal dispersion in the multi-mode gradient-index fiber, the strong coherence of the laser with the multi-mode plastic fiber is destroyed and the image speckle is suppressed. The information points of the disc image (right) can be clearly observed and the imaging quality has been greatly improved. This laser with low-contrast speckle of $4.1 \%$ can be applied for laser illumination in the exhibition industry. 

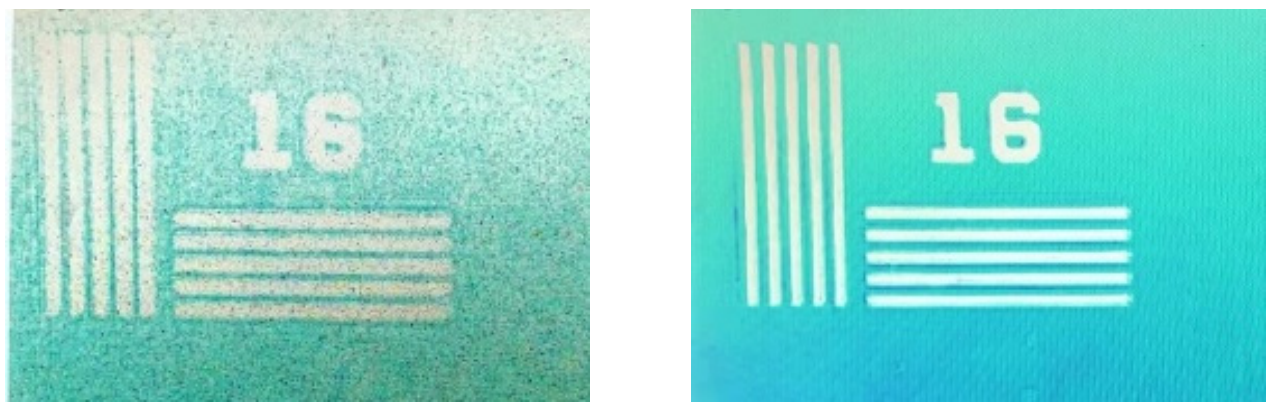

Figure 9. Laser illumination display images before (left) and after (right) speckle suppression with the speckle contrasts of $46.2 \%$ and $4.1 \%$.

\section{Conclusions}

In summary, a low-power laser at $561.5 \mathrm{~nm}$ for exhibit illumination is developed in this paper. The Nd:YAG/PPMgLN module at $561.5 \mathrm{~nm}$ is very simple, compact and efficient. The speckle contrast reduces to $4.1 \%$ by means of a $1000 / 2200$ multi-mode plastic fiber, and the power at $561.5 \mathrm{~nm}$ is $223 \mathrm{~mW}$. The speckle-suppressed Nd:YAG/PPMgLN laser at $561.5 \mathrm{~nm}$ can be used for exhibit illumination due to the low speckle contrast of $4.1 \%$. The results and findings of our laser source experiment can be applied in multiple scenarios and situations in the virtual exhibition industry, such as cultural and historical relic shows, art exhibitions, commercial displays and interactive performance, as well as in conference and exhibition centers, museums, archives and libraries. Relying on this speckle-suppressed laser in this research, exhibitions and shows can provide clear and high-quality visual images and achieve a better exhibition effect to fulfill the needs of the audience. The exhibition effects of art works are enhanced with the application of laser exhibition technology, providing a more immersive experience for audiences.

Author Contributions: Conceptualization, X.L. and H.-Y.L.; methodology, X.L., X.-T.Z. and H.-Y.L.; software, S.-F.B. and T.Y.; validation, X.L., T.Y. and H.-Y.L.; formal analysis, X.L., X.-T.Z., W.-J.S. and H.-Y.L.; investigation, X.L. and X.-T.Z.; resources, X.L. and W.-J.S.; data curation, S.-F.B. and H.-Y.L.; writing-original draft preparation, X.L.; writing - review and editing, X.L. and H.-Y.L.; visualization, T.Y. and S.-F.B.; supervision, H.-Y.L.; funding acquisition, X.L. All authors have read and agreed to the published version of the manuscript.

Funding: This research was funded by the National Social Science Fund of China, grant number 16CGL021.

Institutional Review Board Statement: Not applicable.

Informed Consent Statement: Not applicable.

Data Availability Statement: The data presented in this study are available on request from the corresponding author. The data are not publicly available due to privacy.

Acknowledgments: We are very grateful to the relevant fund of its support and reviewers' valuable comments for this paper.

Conflicts of Interest: The authors declare no conflict of interest.

\section{References}

1. Liu, X.; Seevers, R.; Gu, Z.; Yang, X. Smart MICE: Definitions, foundations and development. In Proceedings of 7th International Conference on Information Science and Control Engineering (ICISCE), Changsha, China, 18-20 December 2020; pp. $1307-1311$.

2. Xiao, L. A conceptual framework for smart MICE ecosystem in China. In Proceedings of the 2017 Euro-Asia Conference on Environment and CSR: Tourism, Society and Education Session (Part I), Hamburg, Germany, 26-27 August 2017; Zhang, Y., Ed.; Wissenschaftlicher Verlag: Berlin, Germany, 2017; pp. 1-10.

3. Bruno, F.; Bruno, S.; Sensi, G.D.; Luchi, M.-L.; Mancuso, S.; Muzzupappa, M. From 3D reconstruction to virtual reality: A complete methodology for digital archaeological exhibit. J. Cult. Herit. 2010, 11, 42-49. [CrossRef]

4. Houston, J.; Beck, W. Design considerations for cinema exhibition using rgb laser illumination. SMPTE. Mot. Imaging J. 2015, 124, 26-41. [CrossRef] 
5. Liang, N. The application of the holographic laser projection in the entertaining performance. In Proceedings of the International Conference on Advanced Materials for Science and Engineering (ICAMSE), Tainan, Taiwan, 12-13 November 2016; pp. 629-631.

6. Blanche, P.-A.; Bablumian, A.; Voorakaranam, R.; Christenson, C.; Lin, W.; Gu, T.; Flores, D.; Wang, P.; Hsieh, W.-Y.; Kathaperumal, M.; et al. Holographic three-dimensional telepresence using large-area photorefractive polymer. Nature 2010, 468, 80-83. [CrossRef] [PubMed]

7. Caggianese, G.; Gallo, L.; Neroni, P. Evaluation of spatial interaction techniques for virtual heritage applications: A case study of an interactive holographic projection. Future Gener. Comp. Syst. 2018, 81, 516-527. [CrossRef]

8. Li, D.; Kelly, D.P.; Sheridan, J.T. Speckle suppression by doubly scattering systems. Appl. Opt. 2013, 52, 8617-8626. [CrossRef]

9. Hepburn, M.S.; Foo, K.Y.; Wijesinghe, P.; Munro, P.R.T.; Chin, L.; Kennedy, B.F. Speckle-dependent accuracy in phase-sensitive optical coherence tomography. Opt. Express 2021, 29, 16950-16968. [CrossRef]

10. Song, Q.; Wang, G.; Zhang, B.; Wang, W.; Wang, M.; Zhang, Q.; Sun, G.; Bo, Y.; Peng, Q. Diode-pumped passively dual-wavelength Q-switched Nd:GYSGG laser using graphene oxide as the saturable absorber. Appl. Opt. 2015, 54, 2688-2692. [CrossRef] [PubMed]

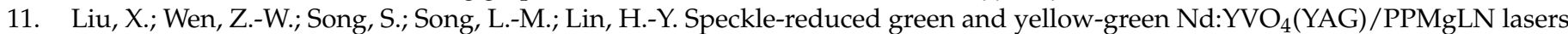
for cinema exhibition industry. Optik 2021, 243, 167427. [CrossRef]

12. Hansen, A.K.; Christensen, M.; Noordegraaf, D.; Heist, P.; Papastathopoulos, E.; Loyo-Maldonado, V.; Jensen, O.B.; Skovgaard, P.M.W. Efficient generation of $1.9 \mathrm{~W}$ yellow light by cascaded frequency doubling of a distributed Bragg reflector tapered diode. Appl. Opt. 2016, 55, 9270-9274. [CrossRef]

13. Hofmann, J.; Blume, G.; Jedrzejczyk, D.; Eppich, B.; Feise, D.; Kreutzmann, S.; Sahm, A.; Paschke, K. Miniaturized diode laser module emitting green light at $532 \mathrm{~nm}$ with a power of more than $900 \mathrm{~mW}$ for next-generation holographic displays. Opt. Rev. 2016, 23, 141-145. [CrossRef]

14. Song, S.; Lin, H.-Y.; Shi, W.-J.; Wen, Z.-W.; Ruan, J.-J.; Liu, H.; Sun, D. Small yellow-green Nd:YAG/PPMgLN laser module at 561.3 nm. Optik 2021, 232, 166557. [CrossRef]

15. Lin, H.; Tan, H.; Miao, J.; Cui, T.; Su, S.; Guo, J. Extra-cavity, widely tunable, continuous wave MgO-doped PPLN optical parametric oscillator pumped with a Nd:YVO 4 laser. Opt. Mater. 2009, 32, 257-260. [CrossRef]

16. Xin, Z.; Hengli, Z.; Yefei, M.; Sihan, S. Efficient methods of green output by second harmonic generation with short pulse broad-band laser. Chin. J. Lasers 2016, 43, 0202003.

17. Zaletelj, K.; Agrež, V.; Slavič, J.; Petkovšek, R.; Boltežar, M. Laser-light speckle formation for deflection-shape identification using digital image correlation. Mech. Syst. Signal Pr. 2021, 161, 107899. [CrossRef]

18. Redding, B.; Choma, M.A.; Cao, H. Speckle-free laser imaging using random laser illumination. Nat. Photonics 2012, 6, 355-359. [CrossRef] [PubMed]

19. Ma, Q.; Xu, C.-Q. Wavelength blending with reduced speckle and improved color for laser projection. Opt. Laser. Eng. 2017, 97, 27-33. [CrossRef]

20. Trisnadi, J.I. Hadamard speckle contrast reduction. Opt. Lett. 2004, 29, 11-13. [CrossRef]

21. Dadabayev, R.; Shabairou, N.; Zalevsky, Z.; Malka, D. A visible light RGB wavelength demultiplexer based on silicon-nitride multicore PCF. Opt. Laser Technol. 2019, 111, 411-416. [CrossRef]

22. Shabairou, N.; Cohen, E.; Wagner, O.; Malka, D.; Zalevsky, Z. Color image identification and reconstruction using artificial neural networks on multimode fiber images: Towards an all-optical design. Opt. Lett. 2018, 43, 5603-5606. [CrossRef]

23. Senarathna, J.; Rege, A.; Li, N.; Thakor, N.V. Laser speckle contrast imaging: Theory, instrumentation and applications. IEEE Rev. Biomed. Eng. 2013, 6, 99-110. [CrossRef]

24. Yoon, Y.; Breshike, C.J.; Kendziora, C.A.; Furstenberg, R.; McGill, R.A. Reduction of speckle noise and mitigation of beam wander in tunable external cavity quantum cascade lasers using rotating diamond/KBr pellet coupled with multimode fiber. Opt. Express 2019, 27, 8011-8020. [CrossRef] [PubMed] 